\title{
EQ-5D em Estudantes Universitários de Medicina - Estudo Observacional
}

\section{EQ-5D Applied to Medical Students - Observational Study}

Cátia Marisa Santos Nunes*, Luiz Miguel Santiago**, Carlos Braz Saraiva***

\section{Pontos-chave:}

A medição da Qualidade de Vida em estudantes é importante para a manutenção da melhor capacidade de aprendizagem.

A utilização de instrumentos validados e adaptados é determinante para a boa execução deste tipo de tarefa. O sexo masculino, a satisfação com a vida social, a satisfação com a vida estudantil e a preocupação com o futuro interferem com o estado de saúde dos alunos.

\section{Resumo}

Introdução: A qualidade de vida $(\mathrm{QV})$ relacionada com a saúde dos jovens estudantes finalistas de Medicina é um tema ainda pouco explorado e que deve ser medido utilizando instrumentos validados, tentando igualmente perceber quais os fatores que a podem influenciar, bem como em que medida.

Objetivo: Estudar a qualidade de vida dos estudantes do $6^{\circ}$ Ano de Medicina, segundo variáveis epidemiológicas.

Metodologia: Estudo observacional, transversal e analítico, realizado em Setembro, na Faculdade de Medicina da Universidade de Coimbra, por aplicação do questionário EQ-5D (Índice e EQ-VAS) a todos os alunos do $6^{\circ}$ Ano de 2013/2014, juntamente com um inquérito epidemiológico sobre variáveis económico-sociais. Realização de estatística descritiva e inferencial.

Resultados: De uma população de 270 alunos de Medicina, foi obtida uma amostra de 186 participantes (69\% sexo feminino) com uma média de 23,6 \pm 1,7 anos de idade. A média do Índice de QV dos estudantes é de 0,88 $\pm 0,15$ e a do EQ-VAS de $83,0 \pm 13,6$. A QV é melhor percebida no sexo masculino (Índice de QV đ̄ 0,92 $\pm 0,13$ vs o $0,86 \pm 0,15 p=0,005$ e EQ-VAS $86,5 \pm 10,9$ vs ๆ $81,1 \pm 14,6 p=0,009$, respetivamente). Tem diferença

\begin{abstract}
Introduction: The health-related quality of life of senior medical students is a yet unexplored subject, that should be assessed by validated tests, so as to learn which factors may contribute to it, and how.

Objective: To study the quality of life of 6th-year medical students, relative to specific epidemiological variables.

Methods: Transversal, observational and analytic study in September 2013, in the Faculty of Medicine of the University of Coimbra, through the distribution of the EQ-5D survey (Index and EQ VAS) to all 6th-year medical students of the class of 2014 , along with an epidemiological survey on socioeconomic variables. Descriptive and inferential statistics were performed.
\end{abstract}

Results: A sample of 186 subjects (69\% female) was obtained from a global population of 270 medical students. The subjects had a mean age of $23.6 \pm 1.7$ years. The mean Quality of Life Index was $0.88 \pm 0.15$ and that of the EQ VAS survey was $83.0 \pm 13.6$. The quality of life is higher in the male gender (Quality of Life Index $0.92 \pm$ 0.13 vs $0.86 \pm 0.15 p=0.005$; EQ VAS 0 o $86.5 \pm 10.9$ vs $081.1 \pm 14.6 p=0.009)$. Statistic differences were found for satisfaction with social life $(0,89 \pm 0,14$ vs 0,78 $\pm 0,15 p<0.001)$, health state perception $(84,1 \pm 12,9$

\footnotetext{
* Aluna Finalista do Mestrado Integrado em Medicina da Faculdade de Medicina da Universidade de Coimbra

** MD, PhD. Consultor, Assistente Graduado Sénior, USF Topázio, Faculdade de Ciências da Saúde da Universidade da Beira Interior

**** Professor Agregado de Psiquiatria da Faculdade de Medicina da Universidade de Coimbra
} 
com significado a satisfação com a vida social $(0,89 \pm$ 0,14 vs $0,78 \pm 0,15 p<0,001$ ), e a perceção do estado de saúde $(84,1 \pm 12,9$ vs 76,2 $\pm 16,2 p=0,005)$, a satisfação na vida estudantil no EQ-5D $(0,89 \pm 0,14$ vs 0,81 \pm $0,17 p=0,008)$. A preocupação com o futuro é diferente no índice EQ-5D $(0,86 \pm 0,15$ vs 0,90 $\pm 0,14 p=0,047)$ e no EQ-VAS $(80,5 \pm 15,2$ vs $85,1 \pm 11,8 p=0,021)$.

Discussão: Sem outros valores para comparar com os presentes percebe-se a importância estratégica de estudar determinantes e influenciadores da QV de estudantes finalistas de Medicina para que possam ser desenhadas as medidas táticas tendentes à melhoria das suas capacidades de estudo e aprendizagem, devendo esta atividade de medida ser realizada regularmente.

Conclusão: Variáveis socio-epidemiológicas como o sexo masculino, a satisfação com a vida social e estudantil e preocupação com o futuro interferem com o estado de saúde dos alunos.

Palavras-chave: Qualidade de vida (QV) relacionada com a saúde; EQ-5D; variáveis epidemiológicas; estudantes.

\section{Introdução}

O EQ-5D fornece uma medida simples e "genérica de medição da qualidade de vida $(\mathrm{QV})$ relacionada com a saúde, que permite gerar um índice representando o valor do estado de saúde de um indivíduo". ${ }^{1}$ É usada em estudos clínicos e observacionais, avaliações económicas de custo-utilidade e pesquisas de saúde populacional, através de um método descritivo onde se incluem cinco domínios ou dimensões e uma escala visual analógica de autoperceção de saúde..$^{1,2}$

Estabelecido em 1987, pelo Grupo EuroQol, a versão portuguesa do questionário EQ-5D, que foi sujeita a procedimentos de tradução e retroversão segundo as normas do grupo, é finalizada em 1998, apresentando uma "boa aceitabilidade, fiabilidade e validade na medição do estado de saúde”.1,2

Verifica-se crescente valorização da medição da QV pelo uso de diferentes instrumentos que permitem verificar a influência de fatores individuais e externos no que é pretendido medir. A informação relativa à QV relacionada com a saúde nos jovens é escassa ${ }^{4-6}$ e muito poucos estudos publicados têm utilizado instrumentos validados que reflitam o conceito multidimensional e subjetivo de QV. ${ }^{3}$ vs 76,2 $\pm 16,2 p=0.005$, ), academic life EQ-5D (0,89 \pm $0,14$ vs $0,81 \pm 0,17 p=0,008)$ and being concerned with the future (EQ-5D $(0,86 \pm 0,15$ vs $0,90 \pm 0,14 p=0,047)$ e no EQ VAS $(80,5 \pm 15,2$ vs $85,1 \pm 11,8 p=0,021)$

Discussion: With no additional data to compare with these findings, the strategic value of studying the variables which influence and determine the quality of life of senior medical students seems clear. A better understanding of these factors may allow the development of measures to improve the studying and learning process. As such, these surveys should be performed regularly.

Conclusions: Socio-epidemiological variables, such as male gender, social and academic life satisfaction and concern with the future, interfere with the students' health.

Keywords: Health-related quality of life; EQ-5D; epidemiological variables; students.

A Universidade é um local onde os estudantes passam muito tempo. Os estudantes universitários são considerados como um grupo populacional de seleção e transição, constituindo um importante "rito de passagem" 4,5 onde os hábitos relacionados com a saúde diferem de outros grupos de população e serão difíceis de mudar mais tarde ${ }^{6}$, sendo o seu estudo relevante. Noutra perspetiva, apesar da QV associada à saúde dos estudantes universitários ser boa ${ }^{5,7}$, estes enfrentam uma variedade de fatores como sobrecarga académica, baixo controlo sobre situações pessoais, dificuldades financeiras, mercado de trabalho competitivo e individualista e perspetivas de vida profissional sombrias, que como problemas futuros, podem comprometer a sua QV.5,6

A Universidade de Coimbra (UC), uma das mais antigas e prestigiadas da Europa, tem arreigadas tradições e forte espírito académico, sendo o elemento chave da vida social estudantil. O Mestrado Integrado em Medicina na UC tem duração de seis anos num total de créditos ECTS de 360,0. $06^{\circ}$ Ano corresponde ao Estágio Orientado e Programado, colocando o académico diretamente em contatos práticos no campo de atuação de trabalho, resultando num período de transição para o futuro da carreira médica, numa fase de elevado stress. ${ }^{7}$ 
A medição da QV em estudantes universitários associada a questões socio-epidemiológicas, será importante por permitir pensar em táticas de ajuda para o objetivo estratégico da melhoria do estado de saúde dos jovens, por parte dos órgãos da Faculdade, da Universidade e da própria academia.

O objetivo deste trabalho é estudar, pela aplicação do EQ$-5 D$, a QV de jovens universitários de medicina portugueses, alunos do $6^{\circ}$ ano da Faculdade de Medicina da Universidade de Coimbra, analisando o impacto de variáveis relacionadas com a vida académica e social, através de um questionário epidemiológico, esperando-se conhecer os determinantes de uma pior qualidade de vida.

\section{Material e Métodos}

Tipo de estudo, participantes e procedimento

Estudo observacional, transversal e analítico, realizado em 25 de Setembro de 2013, na Faculdade de Medicina da Universidade de Coimbra, por aplicação do questionário EQ-5D a todos os alunos do $6^{\circ}$ Ano de 2013/2014 presentes em Seminário, com o consentimento do Docente da aula, previamente concedido. Amostra igual à população. 0 Universo não pôde ser estudado dado haver alunos ausentes do país em estágios opcionais. A participação foi voluntária, anónima e confidencial. Os inquéritos foram recolhidos após o seu preenchimento na saída da aula.

Prévio ao EQ-5D e na mesma folha foi realizado um inquérito epidemiológico sobre variáveis económicosociais constituído por 11 questões de escolha múltipla com os seguintes dados demográficos: idade, sexo, ocupação fora de estudo, residência no período de aulas, ser portador de alguma doença crónica, hábitos tabágicos, hábitos alcoólicos, satisfação com a vida social, satisfação com a vida estudantil, dificuldades financeiras sentidas e preocupação com o futuro profissional.

Foi usada a versão Portuguesa do EQ-5D, que apresenta cinco domínios/dimensões descritivas do estado de saúde: mobilidade, cuidados pessoais, atividades habituais, dor/mal-estar e ansiedade/depressão. Para cada domínio são apresentadas três categorias de resposta: sem problemas (nível 1), alguns problemas (nível 2), problemas severos (nível 3). Torna-se então possível a descrição de $3^{\wedge} 5=243$ estados de saúde. [1,2,8,10] 0 EQ-5D dispõe ainda de uma última pergunta correspondente à perceção do próprio estado de saúde, comparativamente ao seu nível geral de saúde nos 12 meses anteriores ("melhor", "o mesmo", "pior").1,2
Foi também aplicado o EQ-VAS que corresponde a uma escala visual analógica que varia de 0 (pior estado de saúde imaginável) a 100 (melhor estado de saúde imaginável), onde é registada a autoperceção relativamente ao melhor estado de saúde geral dos inquiridos. ${ }^{1,2}$

\section{Análise estatística}

Realizou-se análise estatística descritiva e inferencial com "SPSS software for Windows - version 19.0" (SPSS Inc., Chicago, IL). Foram usados testes paramétricos, test t de student para variáveis não emparelhadas, após verificação da normalidade dos dados, e testes não-paramétricos, U de Mann-whitney e one way ANOVA, considerando diferença estatística para $p<0,05$.

\section{Resultados}

De uma população de 270 alunos de Medicina dos quais 174 são do sexo feminino e 96 do masculino, foram recebidos 186 questionários (proporção de resposta de $64,4 \%)$. Pelo cálculo do tamanho da amostra com uma margem de erro de $5 \%$ e um intervalo de confiança de 95\% (http://www.vsai.pt/amostragem.php), deveria ser estudada uma amostra de $n=159$.

Como podemos observar na tabela 1 , relativa aos dados epidemiológicos, a idade dos inquiridos apresenta uma média de 23,6 \pm 1,7 anos (idades compreendidas entre os 22 e os 32 anos).

Responderam ao questionário 120 raparigas (69,0\% do total de raparigas do curso) e 66 rapazes (68,8\% do total de rapazes do curso). No que respeita à ocupação fora do estudo, 66,1\% dos alunos apresenta uma atividade extracurricular. A maioria residem em casa partilhada $(66,1 \%)$ durante o período de aulas e $10,2 \%$ vivem sozinhos, morando os restantes com a família (23,7\%). Em 16,1\% dos estudantes há doença crónica. Quanto a hábitos tabágicos, a maior percentagem não fuma (79,6\%), 11,3\% fumam episodicamente e 9,1\% fumam regularmente. Têm hábitos alcoólicos frequentes 3,2\% dos respondentes, $82,8 \%$ hábitos ocasionais, nunca tendo bebido 14,0\% da amostra. Há insatisfação com a vida social em 14,0\% dos estudantes e em 13,4\% não há satisfação com a vida estudantil. Não são referidas dificuldades financeiras em $84,9 \%$ da amostra, sendo-o referido em 15,1\%. A preocupação com o futuro é assinalada por 46,2\% dos respondentes, preocupando-se ocasionalmente 53,2\%. Apenas um aluno respondeu nunca ter preocupações. 
Tabela 1: Dados relativos ao questionário epidemiológico

\begin{tabular}{|c|c|c|c|}
\hline Variáveis & & $\mathrm{n}$ & $\%$ \\
\hline \multirow{2}{*}{ Sexo } & Feminino & 120 & 64,5 \\
\hline & Masculino & 66 & 35,5 \\
\hline \multirow{2}{*}{ Ocupação fora do estudo } & Sim & 123 & 66,1 \\
\hline & Não & 63 & 33,9 \\
\hline \multirow{3}{*}{$\begin{array}{l}\text { Residência no período } \\
\text { de aulas }\end{array}$} & Sozinho & 19 & 10,2 \\
\hline & Com a família & 44 & 23,7 \\
\hline & Em casa partilhada & 123 & 66,1 \\
\hline \multirow{2}{*}{$\begin{array}{l}\text { Portador de doença } \\
\text { crónica }\end{array}$} & Sim & 30 & 16,1 \\
\hline & Não & 156 & 83,9 \\
\hline \multirow{3}{*}{ Hábitos tabágicos } & Sim & 17 & 9,1 \\
\hline & Não & 148 & 79,6 \\
\hline & Episódico & 21 & 11,3 \\
\hline \multirow{3}{*}{ Hábitos alcoólicos } & Frequente & 6 & 3,2 \\
\hline & Ocasional & 154 & 82,8 \\
\hline & Nunca & 26 & 14,0 \\
\hline \multirow{2}{*}{$\begin{array}{l}\text { Satisfação com a vida } \\
\text { social }\end{array}$} & Sim & 160 & 86,0 \\
\hline & Não & 26 & 14,0 \\
\hline \multirow{2}{*}{$\begin{array}{l}\text { Satisfação com } \\
\text { actividade estudantil }\end{array}$} & Sim & 161 & 86,6 \\
\hline & Não & 25 & 13,4 \\
\hline \multirow{2}{*}{ Dificuldades financeiras } & Sim & 28 & 15,1 \\
\hline & Não & 158 & 84,9 \\
\hline \multirow{3}{*}{$\begin{array}{l}\text { Preocupação com o } \\
\text { futuro }\end{array}$} & Frequente & 86 & 46,2 \\
\hline & Ocasional & 99 & 53,2 \\
\hline & Nunca & 1 & 0,5 \\
\hline
\end{tabular}

Idade $23,6 \pm 1,7$ anos IC a $95 \%$ 23,3 a 23,8

Segundo os resultados da Tabela 2, relativos ao EQ-5D e EQ-VAS em associação aos dados sociodemográficos, os estudantes relataram uma média de 0,88 \pm 0,15 e 83,0 $\pm 13,6$ (respetivamente) na amostra total. Observou-se que os alunos do sexo masculino apresentam um índice e uma autoperceção de QV relacionada com a saúde superior ao sexo feminino $(0,92 \pm$ 0,13 vs $0,86 \pm 0,15 p=0,005$ e $86,5 \pm 10,9$ vs 81,1 $\pm 14,6 p=0,009$ respetivamente). Tem diferença com significado a satisfação com a vida social $(0,89 \pm 0,14$ vs $0,78 \pm 0,15 p<0,001)$, e a perceção do estado de saúde $(84,1 \pm 12,9$ vs 76,2 $\pm 16,2 p=0,005)$. Os alunos com satisfação na vida estudantil reportam uma melhor QV no índice EQ-5D (0,89 \pm 0,14 vs 0,81 \pm $0,17 p=0,008)$, que no entanto, não tem significado estatístico na autoperceção (83,2 \pm 13,2 vs 81,6 \pm $16,4 p=0,575)$. Também a preocupação com o futuro é significamente relevante no índice EQ-5D $(0,86 \pm$ $0,15 \mathrm{p}=0,047$ vs $0,90 \pm 0,14 p=0,047)$ e no EQ-VAS $(80,5 \pm 15,2$ vs $85,1 \pm 11,8 p=0,021)$.

Relativamente à ocupação fora de estudo, à residência no período de aulas, ao facto de ser portador de doença crónica, aos hábitos tabágicos e alcoólicos e às dificuldades financeiras, verificou-se que não existem diferenças estatísticas significativas. 
Tabela 2: Resultados da amostra total por variáveis do EQ--5D e EQ-VAS em relação aos dados socio-demográficos

\begin{tabular}{|c|c|c|c|c|c|c|}
\hline & & & & & & \\
\hline \multirow[b]{2}{*}{ Variáveis } & & \multirow[b]{2}{*}{$\mathrm{n}$} & \multicolumn{2}{|c|}{ EQ-5D } & \multicolumn{2}{|c|}{ EQ-VAS } \\
\hline & & & $\begin{array}{l}\text { Média } \pm \text { Desvio } \\
\text { Padrão }\end{array}$ & $p$ & $\begin{array}{l}\text { Média } \pm \text { Desvio } \\
\text { Padrão }\end{array}$ & $p$ \\
\hline \multirow{2}{*}{ Sexo } & Feminino & 120 & $0,86 \pm 0,15$ & \multirow{2}{*}{$0,005 *$} & $81,1 \pm 14,6$ & \multirow{2}{*}{$0,009 *$} \\
\hline & Masculino & 66 & $0,92 \pm 0,13$ & & $86,5 \pm 10,9$ & \\
\hline \multirow{2}{*}{ Ocupação fora do estudo } & Sim & 123 & $0,89 \pm 0,15$ & \multirow{2}{*}{0,176} & $83,2 \pm 14,8$ & \multirow{2}{*}{0,841} \\
\hline & Não & 63 & $0,86 \pm 0,14$ & & $82,7 \pm 10,9$ & \\
\hline \multirow{3}{*}{$\begin{array}{l}\text { Residência no período } \\
\text { de aulas }\end{array}$} & Sozinho & 19 & $0,84 \pm 0,16$ & \multirow{3}{*}{0,474} & $78,1 \pm 18,8$ & \multirow{3}{*}{0,192} \\
\hline & Com a família & 44 & $0,89 \pm 0,15$ & & $84,8 \pm 12,6$ & \\
\hline & Em casa partilhada & 123 & $0,89 \pm 0,14$ & & $83,2 \pm 12,9$ & \\
\hline \multirow{2}{*}{$\begin{array}{l}\text { Portador de doença } \\
\text { crónica }\end{array}$} & Sim & 30 & $0,87 \pm 0,14$ & \multirow{2}{*}{0,730} & $80,9 \pm 16,5$ & \multirow{2}{*}{0,352} \\
\hline & Não & 156 & $0,88 \pm 0,15$ & & $83,4 \pm 13,0$ & \\
\hline \multirow{3}{*}{ Hábitos tabágicos } & Sim & 17 & $0,86 \pm 0,15$ & \multirow{3}{*}{0,857} & $78,7 \pm 20,3$ & \multirow{3}{*}{0,107} \\
\hline & Não & 148 & $0,88 \pm 0,14$ & & $84,1 \pm 12,2$ & \\
\hline & Episódico & 21 & $0,89 \pm 0,15$ & & $78,9 \pm 16,0$ & \\
\hline \multirow{3}{*}{ Hábitos alcoólicos } & Frequente & 6 & $0,96 \pm 0,09$ & \multirow{3}{*}{0,126} & $92,3 \pm 3,4$ & \multirow{3}{*}{0,099} \\
\hline & Ocasional & 154 & $0,89 \pm 0,16$ & & $82,2 \pm 14,1$ & \\
\hline & Nunca & 26 & $0,84 \pm 0,15$ & & $85,9 \pm 11,0$ & \\
\hline \multirow{2}{*}{$\begin{array}{l}\text { Satisfação com a vida } \\
\text { social }\end{array}$} & Sim & 160 & $0,89 \pm 0,14$ & \multirow{2}{*}{$<0,001 *$} & $84,1 \pm 12,9$ & \multirow{2}{*}{$0,005^{*}$} \\
\hline & Não & 26 & $0,78 \pm 0,15$ & & $76,2 \pm 16,2$ & \\
\hline \multirow{2}{*}{$\begin{array}{l}\text { Satisfação com } \\
\text { actividade estudantil }\end{array}$} & Sim & 161 & $0,89 \pm 0,14$ & \multirow{2}{*}{$0,008 *$} & $83,2 \pm 13,2$ & \multirow{2}{*}{0,575} \\
\hline & Não & 25 & $0,81 \pm 0,17$ & & $81,6 \pm 16,4$ & \\
\hline \multirow{2}{*}{ Dificuldades financeiras } & Sim & 28 & $0,87 \pm 0,16$ & \multirow{2}{*}{0,713} & $78,9 \pm 19,7$ & \multirow{2}{*}{0,084} \\
\hline & Não & 158 & $0,88 \pm 0,14$ & & $83,7 \pm 12,2$ & \\
\hline & Frequente & 86 & $0,86 \pm 0,15$ & & $80,5 \pm 15,2$ & \\
\hline $\begin{array}{l}\text { Preocupação com o } \\
\text { futuro }\end{array}$ & Ocasional & 99 & $0,90 \pm 0,14$ & $0,047^{*}$ & $85,1 \pm 11,8$ & $0,021 *$ \\
\hline & Nunca & 1 & 1,0 & & 90,0 & \\
\hline Amostra TOTAL & & 186 & $0,88 \pm 0,15$ & & $83,0 \pm 13,6$ & \\
\hline
\end{tabular}

\section{Discussão}

Na recolha da informação para o estudo em causa, o método utilizado foi o preenchimento de um questionário, que permitiu uma maior equidade na obtenção dos resultados. O seu preenchimento esteve dependente da colaboração dos estudantes e da sua presença na aula, impossibilitando o autor de dominar a situação. As variáveis epidemiológicas inquiridas foram selecionadas após eliminação de outras julgadas menos importantes para não se tornar o questionário excessivamente grande e difícil de preencher e, também, para conseguir obter um claro quadro de referências.

Uma vez que a amostra aleatória seria difícil de obter, pela repartição dos alunos do $6^{\circ}$ Ano nos diferentes estágios e Universidades (alunos de mobilidade/Erasmus), bem como, pelo facto de ser importante o preenchimento do inquérito no mesmo dia e à mesma hora, os resultados obtidos são referentes a uma amostra não-probabilística de conveniência, que, no entanto é representativa, pela sua dimensão, do Universo dos alunos. 
Desta forma, os resultados deverão ser cautelosamente analisados encontrando-se como viéses os de oportunidade (aula escolhida para distribuição do questionário), de seleção/amostragem (não é uma amostra aleatória e há uma maior probabilidade da escolha dos alunos que estão presentes na aula), de disponibilidade (alunos de Erasmus/Mobilidade ou os que não estiveram presentes na aula) e de circunstancialismo (escolha do dia e do local da entrega dos questionários).

Por outro lado, existem elementos que podem limitar a discussão dos resultados obtidos, como a falta de trabaIhos publicados acerca do tema em Portugal, as diferentes metodologias dos artigos utilizados e o facto da população universitária em estudo ser restrita a um sub-grupo.

No presente estudo é descrito um grupo de comportamentos dos alunos de $6^{\circ}$ Ano de Medicina da UC, que interferem na QV relacionada com a saúde auto-avaliada e percebida, pela utilização da versão portuguesa do questionário EQ-5D. Os estudantes têm-se apercebido da importância desta temática, nomeadamente, os estudantes de medicina têm demonstrado uma tendência a valorizar mais o estado de saúde que a população geral.5,9

De acordo com a literatura, estudos referem que o processo de formação médica está associada a um declínio nos domínios psicossociais do estado de saúde ${ }^{7}$, bem como um predomínio de problemas do foro psicológico, relativos a dor/desconforto, ansiedade e depressão, podendo estar associados a um mau desempenho académico e profissional. , $5,9^{2}$ No entanto, no estudo efetuado, os estudantes de medicina avaliaram a sua QV relacionada com a saúde como boa, resultado concordante com outros estudos já realizados. ${ }^{4,5,9}$

Comparativamente aos dados registados segundo as normas da população portuguesa, podemos estimar que os alunos apresentam uma QV superior a esta (Índice EQ-5D de 0,758 \pm 0,006 SE-standard error; EQVAS de 74,9 \pm 0,504 SE). ${ }^{8}$ Sabemos que a descrição de um estado de saúde no índice EQ-5D varia entre -0,59 e $1,00^{1}$, verificando-se, no geral, que, as pontuações do EQ-VAS suportam os resultados do Índice EQ-5D. ${ }^{8}$

Quanto ao valor da média da variável em estudo, sexo masculino, pode-se constatar que é mais elevada que a média da amostra total, demonstrando que esta $(p=0,005)$ está associada a uma boa qualidade de vida. Não se verificou diferença entre sexos na análise estatística inferencial no que respeita às diferentes variáveis abordadas, pelo que o estudo foi restringido a uma análise global.
Em relação às áreas EQ-5D e EQ-VAS, sistema descritivo e de autopercepção respectivamente, são consideradas estatisticamente significativas, para determinação de uma boa QV, a satisfação com a vida social e estudantil e a preocupação com o futuro. Contudo, na parte EQ-VAS da variável satisfação com a vida estudantil, não foi encontrado significado estatístico, não interferindo na autopercepção do estado de saúde.

A satisfação com o meio social que envolve os estudantes de medicina é, então um factor favorecedor de bem-estar e de melhoria na QV. Segundo estudos, a satisfação com a vida por parte dos alunos, influencia positivamente a QV, e também é revelado que a vida social tem uma queda durante o último ano de formação académica médica. ${ }^{3}$ Infere-se também que uma menor preocupação com o futuro leva-nos a uma melhor perceção do estado de saúde.

Os resultados nas variáveis ocupação fora de estudo, residência no período de aulas, ser portador de doença crónica, hábitos tabágico e alcoólicos e dificuldades financeiras, não apresentaram significado estatístico, podendo-se inferir que não influenciam a QV relacionada com a saúde dos estudantes de medicina.

Segundo a literatura, determinadas ocupações fora do estudo dos alunos, tais como, trabalho em part-time, afetam a QV relativa à saúde ${ }^{5}$, contudo, não foram encontrados dados estatisticamente significativos que possam associar a presença de uma atividade extracurricular a uma pior qualidade de vida.

A presença de doença crónica é uma das variáveis que não afeta a QV dos jovens universitários, como já foi também observado segundo outros projetos, que é considerada como uma circunstância da vida normal. ${ }^{5}$ Consoante as normas portuguesas da população, a presença de doença crónica está associada a valores mais baixos na avaliação da QV, no entanto, os valores da população universitária em estudo são superiores aos da população geral, constatando-se que os jovens com doença crónica têm melhor QV que os portugueses doentes.

Relativamente aos hábitos, apesar de ser demostrado que ao longo dos anos académicos, beber apresenta um papel central nos eventos sociais e o álcool esteja a aumentar entre os estudantes universitários ${ }^{4}$, observa-se no presente trabalho que a maioria dos inquiridos (82\%) é consumidor ocasional, não demostrando este parâmetro, interferência com a QV dos estudantes. Quanto a hábitos tabágicos, este não são muito prevalentes entre os estudantes inquiridos $(20,4 \%$ 
responderam fumar), não apresentando impacto na QV, todavia estudos revelam que o seu consumo tem aumentado entre a população universitária, associada à gestão do stress e devendo ser um aspeto que necessita de alguma preocupação. ${ }^{4}$

O estudo estabelece uma linha de base para o acompanhamento dos estudantes do $6^{\circ}$ ano de Medicina, para promoção do seu estado de saúde. No entanto, impõe-se a questão: Poderão estes resultados ser verificados noutros anos do curso médico, ou noutras populações jovens universitárias em que o futuro seja diferente após terminar a Licenciatura ou Mestrado Integrado? Para tal novos estudos são importantes.

\section{Referências Bibliográficas}

1. Ferreira, P.L., Ferreira, L.N., Pereira, L.N.. Contributos para a validação da versão portuguesa do EQ- 5D. Acta Médica Portuguesa in press;

2. Cheung, K., Oemar, M., Oppe M., Rabin, R.. User Guide: Basic information on how to use EQ-5D. EuroQol Group 2009:1-24;

3. Paro, H.B.M.S., Morales, N.M.O., Silva, C.H.M., Rezende, C.H.A., Pinto, R.M.C.,Morales R.R., et al. Health-related quality of life of medical student. Medical Educaction 2010; 44:227-235;

4. Vaez, M., Leon, A. P. de, Laflamme, L.. Health-related determinants of perceived quality of life: A comparison between first-year university students and their working peers. Work 26 (2006):167-177;

5. Klemenc-Ketis, Z., Kersnik, J., Eder, K., Colaric, D.. Factors Associated With Health-Related Quality of Life among University Students. Srp Arh Celok Lek. 2011 Mar-Apr;139 (3-4):197-202;

Conflito de Interesses

Os autores declaram não ter conflito de interesses em relação a este artigo.

Endereço para Correspondência:

Cátia Marisa Santos Nunes

Telemóvel: 914853519

Email: catia.santos.nunes @gmail.com

\section{Conclusão}

A QV dos estudantes de $6^{\circ}$ Ano da Faculdade de Medicina da UC foi avaliada como boa. Variáveis socio-epidemiológicas como o sexo masculino, a satisfação com a vida social, a satisfação com a vida estudantil e a preocupação com o futuro interferem com o estado de saúde dos alunos.

Através do conhecimento das pistas associadas a uma pior QV, é importante identificar, sensibilizar e orientar as necessidades especiais dos alunos, para que estratégias de ajuda e melhoria sejam implementadas por parte dos órgãos da Universidade.

6. Vaez, M., Laflamme L.. Health Behaviors, Self-Rated Health, and Quality of life: A Study Among First-Year Swedish University Students. Journal of American college health;51(4):156162;

7. Raj, S.R., Simpson, C.S., Hopman, W.M., Singer, M.A.. Health-related quality of life among final-year medical students. Canadian Medical Association or its licensors, 2000; p. 509-510;

8. Ferreira, L.N., Ferreira, P.L., Pereira, L.N., Oppe, M.. EQ-5D Portuguese population norms. DOI 10.1007/s11136-0130488-4 (acedido a 9 de novembro de 2013);

9. Barbist, M.T., Renn, D., Noisternig, B., Rumpold, G., Höfer, S.. How do medical students value health on the EQ-5D? Evaluation of hypothetical health states compared to the general population. Health and Quality of life Outcomes 2008(6);111;1-6;

10. Ferreira, L.N., Ferreira, P.L., Pereira, L.N., Oppe, M.. The valuation of the EQ-5D in Portugal. Springer Science+Business Media Dordrechet 2013. 


\section{ANEXOS}

ANEXO 1. Questionário Epidemiológico

Questionário Epidemiológico

Num trabalho observacional pretende-se medir a qualidade de vida de jovens universitários. Para tal solicito colaboração pela resposta ao questionário, anónimo e sigiloso abaixo.

Assinale com uma cruz $\mathbf{X}$ cada um dos seguintes itens (exceto o 1, correspondente à idade, que deverá ser respondido em algarismos).

1. Idade:

\section{Sexo:}

$\square$ Feminino

$\square$ Masculino

3. Ocupação fora do estudo:

$\square \operatorname{sim}$

$\square$ Não

4. No período de aulas, reside:

$\square$ Sozinho

$\square$ Com a família

$\square$ Em casa partilhada

5. Portador de alguma doença crónica:

$\square$ Sim

$\square$ Não

6. Hábitos tabágicos:

$\square \operatorname{Sim}$

$\square$ Não

$\square$ Episódico
7. Hábitos alcoólicos:

$\square$ Frequente

$\square$ Ocasional

$\square$ Nunca

8. Está satisfeito com a sua vida social:

$\square \operatorname{Sim}$

$\square$ Não

9. Está satisfeito com a sua actividade estudantil:

$\square \operatorname{Sim}$

$\square$ Não

10. Dificuldades financeiras:

$\square \operatorname{Sim}$

$\square$ Não

11. Preocupação com o futuro:

$\square$ Frequente

$\square$ Ocasional

$\square$ Nunca

Agradeço a participação dos inquiridos e solicito que verifiquem se responderam a todas as questões. 
ANEXO 2. EQ-5D

Avaliação de Ganhos em Saúde | EQ-5D

Assinale com uma cruz (assim $\mathbf{\boldsymbol { \chi }}$ ), um quadrado de cada um dos seguintes grupos, indicando qual das afirmações descreve melhor o seu estado de saúde hoje.

\section{MOBILIDADE}

Não tenho problemas em andar

$\square$ Tenho alguns problemas em andar

$\square$ Tenho de estar de cama

\section{CUIDADOS PESSOAIS}

$\square$ Não tenho problemas em cuidar de mim

$\square$ Tenho alguns problemas a lavar-me ou vestir-me

Sou incapaz de me lavar ou vestir sozinho/a

\section{ACTIVIDADES HABITUAIS}

(ex. trabalho, estudos, actividades domésticas, actividades em família ou de lazer)

Não tenho problemas em desempenhar as minhas actividades habituais

$\square$ Tenho alguns problemas em desempenhar as minhas actividades habituais

$\square$ Sou incapaz de desempenhar as minhas actividades habituais

\section{DOR/MAL ESTAR}

Não tenho dores ou mal estar

$\square$ Tenho dores ou mal estar moderados

Tenho dores ou mal estar extremos

\section{ANSIEDADE/DEPRESSÃO}

Não estou ansioso/a ou deprimido/a

Estou moderadamente ansioso/a ou deprimido/a

$\square$ Estou extremamente ansioso/a ou deprimido/a

COMPARADO COM O MEU NÍVEL GERAL DE SAÚDE DURANTE OS ÚLTIMOS 12 MESES, O MEU ESTADO DE SAÚDE HOJE É:
$\square$ Melhor
0 mesmo
$\square$ Pior

Para ajudar as pessoas a classificarem o seu estado de saúde, desenhámos uma escala (semelhante a um termómetro) na qual o melhor estado de saúde que possa imaginar é marcado por 100 e o pior estado de saúde que possa imaginar é marcado por 0 .

Gostaríamos que indicasse nesta escala qual é hoje, na sua opinião, o seu estado de saúde. Por favor, desenhe uma linha a partir do rectângulo que se encontra à esquerda, até ao ponto da escala que melhor classifica o seu estado de saúde hoje.

O seu estado de saúde hoje

O melhor estado de saúde imaginável

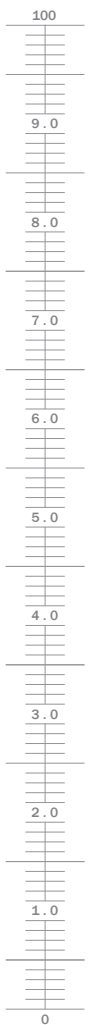

O pior estado de saúde imaginável

Muito obrigado por ter preenchido este questionário,

Copyright (c) Versão Portuguesa, 1997, 1998. Centro de Estudos e Investigação em Saúde da Universidade de Coimbra 